\title{
Cogency, Compactness and Microstructure
}

\section{MARK VOROBEJ}

Department of Philosophy

McMaster University

Hamilton, ON

Canada L8S 4L8

vorobej@mcmaster.ca

The purpose of this note is to respond very briefly to four of the most serious criticisms of my book, A Theory of Argument (Cambridge University Press, 2006) (TA), articulated by Geoff Goddu in a lengthy review that appeared recently in this journal (Vol. 27, 2007, pp. 293-299) (IL).

1. My book defends an account of argument cogency according to which a person $P$ ought to be persuaded by an argument $A$ just in case it's rational for $P$ to believe that (i) each of $A$ 's premises is true, (ii) $A$ 's premise set $S$ is relevant to $A$ 's conclusion, (iii) $S$ provides enough evidence to justify belief in (i.e. to ground) $A$ 's conclusion, and (iv) $A$ is compact. Clause (ii) is redundant in the sense that any argument that satisfies the third (or G condition) will trivially satisfy the second (or R) condition as well. So, Goddu asks, why bother with relevance as a separate condition of cogency ( $I L$, p. 297)?

Relevance can seem unimportant if we focus exclusively on argumentative success. If we understand why it's rational for $P$ to believe that $S$ grounds A's conclusion, then it's pointless to inquire separately whether it's rational for $P$ to believe that $S$ is relevant to that conclusion. But if we're also interested in understanding argumentative failure- the various ways in which and reasons why arguments fail to be cogentthen there's a world of difference between an argument that fails the $G$ condition because it fails the $\mathrm{R}$ condition, and an argument that fails the $\mathrm{G}$ condition despite the fact that it passes the $\mathrm{R}$ condition. Some arguments fail to be cogent because they appeal to irrelevant information. Other arguments fail to be cogent because they appeal to information that is relevant but not substantial enough to justify belief in the conclusion. There's no way of marking this important distinction without invoking (something like) the R condition.

2. An argument $A$ is compact just in case each proper subset of its premise set $S$ provides less evidential support for $A$ 's conclusion than does $S$ itself. Goddu claims that it is again "not clear" why compactness is included as a cogency condition since "surely the inclusion of redundant ... premises does not suddenly make belief in the conclusion irrational” (IL, p. 297). Goddu is correct that it can indeed be rational to

(c) Mark Vorbej. Informal Logic, Vol. 28, No. 3 (2008), pp. 279-281. 
believe the conclusion of a non-compact argument, and I make this point explicitly on page 89 of my text. My view, however, is that a noncompact argument is nonetheless "flawed as an exercise in rational persuasion" (TA, p. 89). Goddu fails to mention this or to discuss my arguments for this position, and so fails to convey the full extent to which my conception of cogency "represents one particularly stringent kind of ideal evidentiary standard” (TA, p. 87).

Curiously, Goddu also worries that the inclusion of compactness as a cogency condition "conflicts" with my stated preference for invulnerable over vulnerable arguments ( $I L$, p. 297). I agree that the conflict is real in the sense that many non-compact arguments are invulnerable, and many compact arguments are vulnerable. But this is simply a reflection of the fact that a multitude of factors contribute, often in conflicting ways, to our robust notion of global, or overall argument strength. (See TA, pages 128 and 254 for just two of a number of discussions of this point.) For reasons explored in my text, vulnerability is indeed a liability - in and of itself, something that detracts from overall argument strength. But Goddu fails to note that the preference for invulnerable arguments holds only ceteris paribus (TA, p. 249), and that this consideration typically matters less than other considerations that bear directly on cogency. In cases of conflict, cogency often trumps other concerns. It's neither surprising nor problematic, then, that cogent arguments exist that are both compact and vulnerable. In fact, every single-premise cogent argument (with a relevant premise set) will have these two properties.

3. The second half of my text develops an account of argument diagraming that concentrates on representing the internal microstructure of arguments, as conceived by the authors of those arguments. Diagrams, in other words, capture authorial beliefs about relevance relations. Goddu predicts that "anyone who thinks we ought to be diagraming the actual evidential relations rather than the evidential relations as conceived by the author will once again be frustrated" (IL, p. 297).

It is true that my account has a very specific focus. This brings clarity to the project but, for some, the focus may seem excessively narrow. Notice, however, that neither the diagraming apparatus (with the exception of squigglification) nor the definitions of convergent, linked and hybrid arguments make any reference whatsoever to the epistemic notion of belief. Therefore, for those so inclined, it's possible simply to appropriate all of this material while stipulating anew that the goal of argument diagraming is to capture actual relevance relations. So there's no need for frustration on this score.

Of course, this doesn't alter the fact that much of my discussion grapples with the challenges involved in working with texts in an attempt to ascertain the beliefs of authors and audience members. These challenges are both fascinating and difficult to avoid. Suppose you're interested in discovering actual relevance relations. Once you encounter even a moderately controversial argument, however, you're likely to become embroiled in disagreements over whether and, if so, how certain 
premises are actually relevant to a certain conclusion. To understand and to benefit from any such disagreement, you need to understand what certain individuals believe about the relevance relations in question, and why they hold those beliefs. In no time at all, you're struggling with some of the central problems addressed within A Theory of Argument.

4. Goddu's harshest criticism is also his most puzzling. Essentially, he wonders why we should bother with the whole enterprise of argument diagraming. Specifically, he charges that my account "provides no reason why determining an argument's microstructure is at all important for those concerned with the presentation and evaluation of arguments" (IL, p. 298). Since Goddu acknowledges that "understanding how premises ground (or fail to ground) [a] conclusion" is "one of the most important issues of argument theory” (IL, p. 298), let me respond to his criticism as follows.

You can't determine whether an argument is grounded without determining whether its premise set is relevant to its conclusion. If you determine that the premise set is relevant, you ought to be able to explain how it is relevant. This matters because argument diagraming highlights the fact that many structural possibilities exist. And skill in argument diagraming allows you to articulate these various options with great clarity. Furthermore, the search for a relevant microstructural option often complements our search for unexpressed premises, and often suggests alternative (and perhaps unanticipated) semantic interpretations of the argument's propositional components. Clearly, these considerations can significantly affect our judgment about the strength of the argument's (alleged) grounding relation. So argument diagraming helps in determining whether and, if so, how arguments are grounded.

If you determine that the premise set is not relevant, then you are committed to the claim that none of the various structural options is defensible. Skill in argument diagraming allows you to engage in a constructive discussion of this topic with an interlocutor. To make a credible charge of irrelevance against an author, for example, you need to appreciate how that individual conceives of the microstructure of her own argument. And as I stress throughout my text, this kind of careful and precise discussion about microstructural matters may generate surprising results. Most dramatically, it may bring to light a microstructure that transforms (what you thought was) a weak argument into a grounded argument. Microstructural discussions can therefore assist us greatly in identifying those arguments by which we ought to be persuaded. 